KEK-TH-777

hep-th/0107101

\title{
Comma Vertex and String Field Algebra
}

\author{
Kazuyuki Furuuchi and Kazumi Okuyama \\ Theory Group, KEK, Tsukuba, Ibaraki 305-0801, Japan \\ furuuchi@post.kek.jp, kazumi@post.kek.jp
}

\begin{abstract}
We study the matter part of the algebra of open string fields using the 3-string vertex over the sliver state, which we call "comma vertex". By generalizing this comma vertex to the $N$-string overlap, we obtain a closed form of the Neumann coefficients in the $N$-string vertex and discuss its relation to the oscillator representation of wedge states.
\end{abstract}

July 2001 


\section{Introduction}

Recently, the cubic open string field theory [1] around the tachyon vacuum, or the "vacuum string field theory" [2, 3] was proposed. This theory contains no physical open string excitation and is conjectured to describe the appearance of closed strings after the open string tachyon condensation. The matter part of the solution of the classical equation of motion around this vacuum is given by projection elements in the star algebra of open string fields [1].

As an example of projection, there is a state called sliver [6], which is special in a sense that this state can be defined in an arbitrary boundary CFT. In the case of flat background a projection was also constructed in the oscillator formalism [0] and was identified with the sliver with numerical evidence [4]. To construct projections more systematically, it is important to find a matrix representation of open string field [7, 8, 9] (see [10, 11, 12, 13] for related issues). The essential ingredient behind this matrix representation is the so-called "comma" form of the overlap vertex [14, 15, 16]. Interestingly, if we perform a Bogoliubov transformation to make the sliver state to be a new Fock vacuum, the 3-string vertex takes a comma form [5]. In this respect the sliver is a key to the structure of the string field algebra. In this paper, we study the properties of the overlap vertices constructed over the sliver state, and discuss some applications.

This paper is organized as follows: In section 2, we consider the 3 -string vertex over the sliver state. In section 3, we analyze the structure of string field algebra using this vertex. In section 4, we construct the oscillator representation of wedge states and find a closed form of the Neumann coefficient of $N$-string vertex. Section 5 is devoted to the discussions.

\section{Comma Vertex}

In this section, we review the 3 -string vertex constructed over the sliver state [5]. In the following, we will call this vertex the "comma vertex" [14, 15, 16].

\section{$2.1 \quad 3$-string Neumann coefficient}

The matter part of the 3-string vertex in zero-momentum sector is given by 17, 18, 19

$$
\left|V_{3}\right\rangle=\mathcal{N}_{V_{3}}^{26} \exp \left(-\frac{1}{2} \sum_{\substack{r, s=1,2,3 \\ m, n \geq 1}} a_{m}^{r \dagger} V_{3 m n}^{r s} a_{n}^{s \dagger}\right)|0\rangle_{123} .
$$

$\mathcal{N}_{V_{3}}$ is a normalization constant. In this paper we concentrate on zero-momentum sector but algebraic relations we will obtain are the same if we consider whole sector [5, 4, 8]. The identity string field $|I\rangle$ and the reflector $\langle R(1,2)|$ is given by (up to normalization)

$$
|I\rangle=e^{-\frac{1}{2} a^{\dagger} C a^{\dagger}}|0\rangle, \quad\langle R(1,2)|={ }_{12}\langle 0| e^{-a_{1} C a_{2}},
$$


where $C_{n m}=(-1)^{n} \delta_{n m}$. We define star product of string fields as

$$
|A \star B\rangle_{1}={ }_{3}\left\langle\left. r(A)\right|_{2}\left\langle r(B)|| V_{3}\right\rangle_{123},\right.
$$

where

$$
{ }_{1}\langle r(A)|=\langle R(1,2) \mid A\rangle_{2} .
$$

As preliminaries, we list some useful formulas satisfied by the Neumann coefficients $V_{3}^{r s}$ in the 3 -string vertex. We introduce the matrices $X, Y, Z$ by

$$
X=C V_{3}^{11}, \quad Y=C V_{3}^{12}, \quad Z=C V_{3}^{21} .
$$

It can be shown that these matrices commute with each other and satisfy the following relations:

$$
\begin{aligned}
& X+Y+Z=1, \\
& X^{2}+Y^{2}+Z^{2}=1, \quad Y Z=X^{2}-X, \\
& Y^{3}+Z^{3}=2 X^{3}-3 X^{2}+1, \\
& C X C=X, \quad C Y C=Z .
\end{aligned}
$$

Using these matrices, one can construct the orthogonal projections $L$ and $R$ U

$$
L=\frac{Y(1-T X)+T Z^{2}}{(1+T)(1-X)}, \quad R=\frac{Z(1-T X)+T Y^{2}}{(1+T)(1-X)},
$$

which satisfy

$$
L+R=1, \quad L^{2}=L, \quad R^{2}=R, \quad L R=0, \quad L=C R C .
$$

Here $T$ is defined by

$$
T=\frac{1}{2 X}(1+X-\sqrt{(1-X)(1+3 X)}),
$$

and satisfies

$$
X T^{2}-(1+X) T+X=0 .
$$

This matrix $T$ appears in the sliver state [5, []

$$
|\Xi\rangle=\mathcal{N}_{\infty}^{26} \exp \left(-\frac{1}{2} a^{\dagger} C T a^{\dagger}\right)|0\rangle,
$$

which satisfies $|\Xi\rangle \star|\Xi\rangle=|\Xi\rangle$. The matrices $Y$ and $Z$ are decomposed into $L$ and $R$ as

$$
\begin{aligned}
& Y=L(1-T X)+R X(T-1)=(1-T X)(L-T R), \\
& Z=L X(T-1)+R(1-T X)=(1-T X)(R-T L) .
\end{aligned}
$$

\footnotetext{
${ }^{1}$ In [7], $L$ and $R$ were denoted $\rho_{1}$ and $\rho_{2}$, respectively.
} 


\subsection{Vertex over the Sliver}

We introduce new oscillators $s$ which annihilate the sliver by performing a Bogoliubov transformation from the original oscillators $a$

$$
\begin{gathered}
s=\frac{1}{\sqrt{1-T^{2}}}\left(a+C T a^{\dagger}\right), \quad s^{\dagger}=\frac{1}{\sqrt{1-T^{2}}}\left(a^{\dagger}+C T a\right), \\
s|\Xi\rangle=0 .
\end{gathered}
$$

The 3-string vertex in this new basis (up to normalization) turns out to be [5]

$$
\left|V_{3}\right\rangle=\exp \left(-\sum_{r=1}^{3} s_{r}^{\dagger} C L s_{r+1}^{\dagger}\right)|\Xi\rangle_{123}=\exp \left(-\frac{1}{2} \sum_{r, s=1}^{3} s_{r}^{\dagger} \widehat{V}_{3}^{r s} s_{s}^{\dagger}\right)|\Xi\rangle_{123},
$$

where $\widehat{V}_{3}^{r s}$ is given by

$$
C \widehat{V}_{3}=\left(1-C V_{3} T\right)^{-1}\left(C V_{3}-T\right)=\left(\begin{array}{ccc}
0 & L & R \\
R & 0 & L \\
L & R & 0
\end{array}\right)
$$

The identity string field and the reflector in this new basis are found to be

$$
|I\rangle=e^{-\frac{1}{2} s^{\dagger} C s^{\dagger}}|\Xi\rangle, \quad\langle R(1,2)|={ }_{12}\langle\Xi| e^{-s_{1} C s_{2}} .
$$

Using the projections $L$ and $R$, we can define the splitting of $s$ into two parts

$$
s=\sum_{\alpha} s_{L \alpha} e_{\alpha}+s_{R \alpha} f_{\alpha}
$$

where $e_{\alpha}$ and $f_{\alpha}$ are the orthonormal basis of the eigenspace of $L$ and $R$ :

$$
\begin{gathered}
e_{\alpha} \cdot e_{\beta}=f_{\alpha} \cdot f_{\beta}=\delta_{\alpha \beta}, \quad e_{\alpha} \cdot f_{\beta}=0, \quad f_{\alpha}=-C e_{\alpha}, \\
L e_{\alpha}=e_{\alpha}, \quad R e_{\alpha}=0, \quad L f_{\alpha}=0, \quad R f_{\alpha}=f_{\alpha} \\
L=\sum_{\alpha} e_{\alpha} e_{\alpha}^{T}, \quad R=\sum_{\alpha} f_{\alpha} f_{\alpha}^{T}
\end{gathered}
$$

Since $s_{L}$ and $s_{R}$ commute,

$$
\left[s_{L \alpha}, s_{L \beta}^{\dagger}\right]=\left[s_{R \alpha}, s_{R \beta}^{\dagger}\right]=\delta_{\alpha \beta}, \quad\left[s_{L \alpha}, s_{R \beta}^{\dagger}\right]=0,
$$

the Hilbert space factorizes into the Fock spaces of $s_{L}$ and $s_{R}$

$$
\mathcal{A}_{\text {str }}=\mathcal{H}_{L} \otimes \mathcal{H}_{R}
$$

In terms of $s_{L, R}$, the 3-string vertex takes a simple form ("comma" form)

$$
\left|V_{3}\right\rangle=\exp \left(s_{1 R}^{\dagger} s_{2 L}^{\dagger}+s_{2 R}^{\dagger} s_{3 L}^{\dagger}+s_{3 R}^{\dagger} s_{1 L}^{\dagger}\right)|\Xi\rangle_{123},
$$

where

$$
s_{L}^{\dagger} s_{R}^{\dagger}=\sum_{\alpha} s_{L \alpha}^{\dagger} s_{R \alpha}^{\dagger} .
$$

The identity string field and the reflector become

$$
|I\rangle=e^{s_{R}^{\dagger} s_{L}^{\dagger}}|\Xi\rangle, \quad\langle R(1,2)|={ }_{12}\langle\Xi| e^{s_{1 R} s_{2 L}+s_{2 R} s_{1 L}} .
$$




\subsection{On the Structure of the Vertex over the Sliver}

The vanishing of the diagonal components of $\widehat{V}_{3}$ in $(\widehat{2.20})$ is simply explained from the identity $|\Xi\rangle \star|\Xi\rangle=|\Xi\rangle$ written in terms of the s-oscillators [0]. The identities $L R=0$ and $R L=0$, which seem somewhat accidental from the complicated expression (2.10), also simply follow from $|\Xi\rangle \star|I\rangle=|\Xi\rangle$ and $|I\rangle \star|\Xi\rangle=|\Xi\rangle$ in the $s$-oscillator basis:

$$
\begin{aligned}
(|\Xi\rangle \star|I\rangle)_{1} & ={ }_{3}\left\langle\left.\Xi\right|_{2}\left\langle I|| V_{3}\right\rangle_{123}\right. \\
& ={ }_{3}\left\langle\left.\Xi\right|_{2}\left\langle\Xi\left|\exp \left(-\frac{1}{2} s_{2} C s_{2}\right) \exp \left(-\frac{1}{2} \sum_{r, s=1}^{3} s_{r}^{\dagger} \widehat{V}_{3}^{r s} s_{s}^{\dagger}\right)\right| \Xi\right\rangle_{123}\right. \\
& ={ }_{2}\left\langle\Xi\left|\exp \left(-\frac{1}{2} s_{2} C s_{2}\right) \exp \left(-s_{1}^{\dagger} \widehat{V}^{12} s_{2}^{\dagger}\right)\right| \Xi\right\rangle_{12} \\
& =\exp \left(-\frac{1}{2} s_{1}^{\dagger} \widehat{V}^{12} C \widehat{V}^{21} s_{1}^{\dagger}\right)|\Xi\rangle_{1},
\end{aligned}
$$

where use has been made for the formula

$$
\begin{aligned}
& \left\langle 0\left|\exp \left(\lambda_{i} a_{i}-\frac{1}{2} P_{i j} a_{i} a_{j}\right) \exp \left(\mu_{i} a_{i}^{\dagger}-\frac{1}{2} Q_{i j} a_{i}^{\dagger} a_{j}^{\dagger}\right)\right| 0\right\rangle \\
& \quad=\operatorname{det} K^{-1 / 2} \exp \left(\mu K^{-1} \lambda-\frac{1}{2} \lambda Q K^{-1} \lambda-\frac{1}{2} \mu K^{-1} P \mu\right), \quad K \equiv 1-P Q .
\end{aligned}
$$

From eq.(2.31) we observe $|\Xi\rangle \star|I\rangle=|\Xi\rangle$ implies $\widehat{V}^{12} C \widehat{V}^{21}=0$, which is equivalent to $L R=0$. Similarly, $R L=0$ can be understood from $|I\rangle \star|\Xi\rangle=|\Xi\rangle$.

We briefly comment on other identities in (2.11). $\widehat{V}_{3}$ can be diagonalized in the $r, s$ indices by a matrix $\mathcal{O}$ satisfying $\mathcal{O}^{-1}=\mathcal{O}^{\dagger}$ :

$$
\widehat{V}_{3}=\mathcal{O}^{-1} \widehat{V}_{D} \mathcal{O}
$$

with

$$
\widehat{V}_{D} \equiv\left(\begin{array}{ccc}
C & 0 & 0 \\
0 & \Lambda & 0 \\
0 & 0 & \bar{\Lambda}
\end{array}\right), \quad \mathcal{O}=\frac{1}{\sqrt{3}}\left(\begin{array}{ccc}
1 & 1 & 1 \\
\omega & \bar{\omega} & 1 \\
\bar{\omega} & \omega & 1
\end{array}\right) .
$$

Here $\omega \equiv \exp (2 \pi i / 3) . \widehat{V}_{D}^{11}=C$ follows because this component represents identity string field type overlap condition (see (2.2) and (2.21)). From (2.33) and (2.34), we obtain

$$
\begin{aligned}
& C=\widehat{V}_{3}^{12}+\widehat{V}_{3}^{21}, \\
& \Lambda=\bar{\omega} \widehat{V}_{3}^{12}+\omega \widehat{V}_{3}^{21}, \\
& \bar{\Lambda}=\omega \widehat{V}_{3}^{12}+\bar{\omega} \widehat{V}_{3}^{21},
\end{aligned}
$$

and $C=\widehat{V}_{3}^{12}+\widehat{V}_{3}^{21}$ is equivalent to $L+R=1$. Notice that $L^{2}=L$ and $R^{2}=R$ follow from $L+R=1$ and $L R=R L=0$.

Finally the equation $L=C R C$ follows from the property of the original vertex $C V^{12}=$ $V^{21} C$, since $C$ and $T$ which appear in the Bogoliubov transformation (2.17) commute with C. $C V^{12}=V^{21} C$ describes a property of the vertex under the string worldsheet orientation reversal. 


\section{$3 \quad$ String Field Algebra}

In this section, we discuss the structure of the string field algebra $\mathcal{A}_{\text {str }}$. The results in this section were essentially obtained in [9] using a different representation of the vertex.

Strictly speaking, $\mathcal{A}_{\text {str }}$ in this section should be understood as the zero-momentum subalgebra of string field algebra, since the sliver state does not carry momentum. However, the most of the following discussions can be applied to the whole algebra by replacing the sliver state by the $D$-instanton state, since the Neumann coefficients including the zero-modes satisfy the same relations as those without zero-modes [5, 4, 8].

\subsection{String Field Oscillators}

First, we construct the string field oscillators $A_{\alpha}, A_{\alpha}^{\dagger}[9]$ which satisfy the canonical commutation relations

$$
\left[A_{\alpha}, A_{\beta}^{\dagger}\right]_{\star}=\delta_{\alpha \beta}|I\rangle
$$

To find the explicit expression of the string field oscillators, it is convenient to introduce the coherent states

$$
\begin{aligned}
\left|z_{L}, z_{R}\right\rangle & =\exp \left(z_{L} s_{L}^{\dagger}+z_{R} s_{R}^{\dagger}\right)|\Xi\rangle, \\
I\left(z_{L}, z_{R}\right) & =\exp \left(z_{L} s_{L}^{\dagger}+z_{R} s_{R}^{\dagger}\right)|I\rangle .
\end{aligned}
$$

The multiplication rules of these coherent states are found to be

$$
\begin{aligned}
\left|z_{L}, z_{R}\right\rangle \star\left|w_{L}, w_{R}\right\rangle & =e^{z_{R} w_{L}}\left|z_{L}, w_{R}\right\rangle, \\
I\left(z_{L}, z_{R}\right) \star\left|w_{L}, w_{R}\right\rangle & =e^{z_{R} w_{L}}\left|z_{L}+w_{L}, w_{R}\right\rangle, \\
\left|z_{L}, z_{R}\right\rangle \star I\left(w_{L}, w_{R}\right) & =e^{z_{R} w_{L}}\left|z_{L}, z_{R}+w_{R}\right\rangle, \\
I\left(z_{L}, z_{R}\right) \star I\left(w_{L}, w_{R}\right) & =e^{z_{R} w_{L}} I\left(z_{L}+w_{L}, z_{R}+w_{R}\right) .
\end{aligned}
$$

Using (3.7), we can check that the string fields

$$
A_{\alpha}=s_{R \alpha}^{\dagger}|I\rangle, \quad A_{\alpha}^{\dagger}=s_{L \alpha}^{\dagger}|I\rangle
$$

satisfy the relation (3.1). Since the sliver state is annihilated by the string field oscillators

$$
A_{\alpha} \star|\Xi\rangle=|\Xi\rangle \star A_{\alpha}^{\dagger}=0,
$$

the sliver can be identified with a projection onto the Fock vacuum $|0\rangle\rangle$ of the string field oscillators:

$$
|\Xi\rangle=|0\rangle\rangle\langle\langle 0|
$$

This identification is justified by the fact that the sliver is naturally factorized into the vacuum of $s_{L}$ and $s_{R}$ :

$$
|\Xi\rangle=\left|\Xi_{L}\right\rangle \otimes\left|\Xi_{R}\right\rangle
$$


where

$$
s_{L \alpha}\left|\Xi_{L}\right\rangle=0, \quad s_{R \alpha}\left|\Xi_{R}\right\rangle=0
$$

\subsection{Matrix Representation of String Fields}

In this subsection we construct a mapping between open string fields and matrices. Since the identity string field has the form $|I\rangle=e^{s_{L}^{\dagger} s_{R}^{\dagger}}|\Xi\rangle$, the string field oscillators (3.8) can be rewritten as

$$
\begin{aligned}
& A_{\alpha}=s_{L \alpha}|I\rangle=s_{R \alpha}^{\dagger}|I\rangle, \\
& A_{\alpha}^{\dagger}=s_{L \alpha}^{\dagger}|I\rangle=s_{R \alpha}|I\rangle .
\end{aligned}
$$

Using (3.7) recursively, we can show the following relations:

$$
\begin{aligned}
A_{\alpha_{1}} \star \cdots \star A_{\alpha_{k}} & =s_{L \alpha_{1}} \cdots s_{L \alpha_{k}}|I\rangle=s_{R \alpha_{1}}^{\dagger} \cdots s_{R \alpha_{k}}^{\dagger}|I\rangle, \\
A_{\alpha_{1}}^{\dagger} \cdots \star A_{\alpha_{k}}^{\dagger} & =s_{L \alpha_{1}}^{\dagger} \cdots s_{L \alpha_{k}}^{\dagger}|I\rangle=s_{R \alpha_{1}} \cdots s_{R \alpha_{k}}|I\rangle, \\
{\left[A_{\alpha}, A_{\beta}^{\dagger}\right]_{\star} } & =\left[s_{L \alpha}, s_{L \beta}^{\dagger}\right]|I\rangle=\left[s_{R \alpha}, s_{R \beta}^{\dagger}\right]|I\rangle .
\end{aligned}
$$

Therefore, the identity string field defines a morphism between worldsheet oscillators $s_{L, R}$ and the string field oscillators. This induces the following isomorphism between the Fock spaces:

$$
\mathcal{H}_{L} \cong \mathcal{H}_{s t r}, \quad \mathcal{H}_{R} \cong \mathcal{H}_{s t r}^{*}
$$

where $\mathcal{H}_{\text {str }}$ is the Fock space of string field oscillators. Finally we have the identification between open string fields and the matrices acting on $\mathcal{H}_{s t r}$ :

$$
\mathcal{A}_{\text {str }}=\mathcal{H}_{L} \otimes \mathcal{H}_{R}=\mathcal{H}_{\text {str }} \otimes \mathcal{H}_{\text {str }}^{*}=\operatorname{End}\left(\mathcal{H}_{\text {str }}\right)
$$

We can construct this mapping in a more explicit way. Using the morphism (3.16), the star exponential of string field oscillators turns out to be

$$
e_{\star}^{z_{L} A^{\dagger}}=e^{z_{L} s_{L}^{\dagger}}|I\rangle, \quad e_{\star}^{z_{R} A}=e^{z_{R} s_{R}^{\dagger}}|I\rangle .
$$

From (3.5), (3.6) and (3.20), we can see that the coherent states of $s_{L, R}$ and $A_{\alpha}$ are related as

$$
\left.\left|z_{L}, z_{R}\right\rangle=e^{z_{L} s_{L}^{\dagger}}|I\rangle \star|\Xi\rangle \star e^{z_{R} s_{R}^{\dagger}}|I\rangle=e^{z_{L} A^{\dagger}}|0\rangle\right\rangle\left\langle\left\langle 0\left|e^{z_{R} A}=\right| z_{L}\right\rangle\right\rangle\left\langle\left\langle z_{R}\right| .\right.
$$

Note that this relation is consistent with the trace

$$
\operatorname{Tr}\left(\left|z_{L}, z_{R}\right\rangle\right)=\left\langle I \mid z_{L}, z_{R}\right\rangle=e^{z_{L} z_{R}} .
$$

By expanding (3.21) in terms of $z_{L, R}$, we find that the occupation number state of $s_{L, R}$ corresponds to the matrix element between the number states of string field oscillators:

$$
\left.|n, m\rangle=\prod_{\alpha, \beta} \frac{s_{L \alpha}^{\dagger n_{\alpha}} s_{R \beta}^{\dagger m_{\beta}}}{\sqrt{n_{\alpha} ! m_{\beta} !}}|\Xi\rangle=\prod_{\alpha} \frac{A_{\alpha}^{\dagger n_{\alpha}}}{\sqrt{n_{\alpha} !}}|0\rangle\right\rangle\left\langle\left\langle 0\left|\prod_{\beta} \frac{A_{\alpha}^{m_{\beta}}}{\sqrt{m_{\beta} !}}=\right| n\right\rangle\right\rangle\langle\langle m| .
$$


We can see that the number states behave exactly as matrix elements:

$$
\begin{aligned}
|n\rangle\rangle\langle\langle m|\star| k\rangle\rangle\langle\langle l| & \left.=\delta_{m, k}|n\rangle\right\rangle\langle\langle l|, \\
\operatorname{Tr}(|n\rangle\rangle\langle\langle m|) & =\delta_{n, m} .
\end{aligned}
$$

Now we can construct a mapping between string fields and matrices as follows. Since every state can be expanded in terms of the number basis of $s_{L, R}$, every string field can be written as a matrix:

$$
\left.|\Psi\rangle=\sum_{n, m} \Psi_{n, m}|n, m\rangle=\sum_{n, m} \Psi_{n, m}|n\rangle\right\rangle\langle\langle m| .
$$

Especially, the identity string field corresponds to the identity matrix

$$
\left.|I\rangle=e^{s_{L}^{\dagger} s_{R}^{\dagger}}|\Xi\rangle=\sum_{n}|n, n\rangle=\sum_{n}|n\rangle\right\rangle\langle\langle n|
$$

Note that the 3-string vertex can also be written in terms of the number basis

$$
\left.\left|V_{3}\right\rangle_{123}=\sum_{l, m, n}|l\rangle\right\rangle\left\langle\left\langle\left. m\right|_{1} \otimes \mid m\right\rangle\right\rangle\left\langle\left\langle\left. n\right|_{2} \otimes \mid n\right\rangle\right\rangle\left\langle\left\langle\left. l\right|_{3} .\right.\right.
$$

We can see that this vertex represents the matrix multiplication.

\section{Wedge States and $N$-string Vertices}

In this section, we first construct oscillator representations of wedge states, which were originally defined as a class of surface states in [6]. Then we present a closed form of the Neumann coefficients in the $N$-string vertex and discuss their relation to the wedge states.

\subsection{Oscillator Representation of Wedge States}

We define the $n^{\text {th }}$ wedge state $|n\rangle_{w}$ by [6]

$$
|n\rangle_{w} \equiv(|0\rangle)_{\star}^{n-1}
$$

In this subsection, we will find an oscillator representation of the wedge state under the following ansatz 2

$$
|n\rangle_{w}=\mathcal{N}_{n}^{26} \exp \left(-\frac{1}{2} a^{\dagger} C T_{n} a^{\dagger}\right)|0\rangle .
$$

The recursion relations for $\mathcal{N}_{n}$ and $T_{n}$ follow from the relation $|n+1\rangle_{w}=|n\rangle_{w} \star|0\rangle$ :

$$
\begin{aligned}
T_{n+1} & =\frac{X\left(1-T_{n}\right)}{1-X T_{n}} \\
\mathcal{N}_{n+1} & =\mathcal{N}_{n} \mathcal{N}_{V_{3}} \operatorname{det}\left(1-X T_{n}\right)^{-\frac{1}{2}}
\end{aligned}
$$

\footnotetext{
${ }^{2}$ It was informed to us by B. Zwiebach that the oscillator representation of wedge states has been obtained independently by A. Sen.
} 
In the above we have assumed that $T_{n}$ commutes with $X, Y$ and $Z$ which will appear to hold consistently when the explicit form of $T_{n}$ is obtained. In order for the wedge state to converge in the limit $n \rightarrow \infty$, we should set the normalization $\mathcal{N}_{V_{3}}$ of the 3 -string vertex to be

$$
\mathcal{N}_{V_{3}}=\operatorname{det}(1-X T)^{\frac{1}{2}}
$$

By rewriting the recursion relations using (2.13)

$$
\begin{aligned}
\frac{1}{T_{n+1}-T}-\frac{T}{1-T^{2}} & =-\frac{1}{T}\left(\frac{1}{T_{n}-T}-\frac{T}{1-T^{2}}\right), \\
\frac{\mathcal{N}_{n+1}}{\mathcal{N}_{n}} & =\operatorname{det}\left(-\frac{1}{T} \frac{T_{n+1}-T}{T_{n}-T}\right)^{\frac{1}{2}},
\end{aligned}
$$

and putting the initial value $T_{2}=0, \mathcal{N}_{2}=1$, we find

$$
\begin{aligned}
T_{n} & =\frac{T+(-T)^{n-1}}{1-(-T)^{n}} \\
\mathcal{N}_{n} & =\operatorname{det}\left[\frac{1-T^{2}}{1-(-T)^{n}}\right]^{\frac{1}{2}} .
\end{aligned}
$$

Note that the normalization of the identity $\mathcal{N}_{1}$ and the 3-string vertex $\mathcal{N}_{V_{3}}$ satisfy the relation found in [8]:

$$
\mathcal{N}_{1} \mathcal{N}_{V_{3}}=\operatorname{det}(1-X)^{\frac{1}{2}}
$$

Note also that $\mathcal{N}_{V_{3}}$ is equal to $\mathcal{N}_{3}$. The final expression of the wedge state is

$$
|n\rangle_{w}=\operatorname{det}\left[\frac{1-T^{2}}{1-(-T)^{n}}\right]^{\frac{26}{2}} \exp \left(-\frac{1}{2} a^{\dagger} C \frac{T+(-T)^{n-1}}{1-(-T)^{n}} a^{\dagger}\right)|0\rangle .
$$

In this normalization, the sliver state which can be defined as a limit of the wedge states [6] becomes

$$
|\Xi\rangle=\lim _{n \rightarrow \infty}|n\rangle_{w}=\operatorname{det}\left(1-T^{2}\right)^{\frac{26}{2}} \exp \left(-\frac{1}{2} a^{\dagger} C T a^{\dagger}\right)|0\rangle .
$$

Here we assumed

$$
\lim _{n \rightarrow \infty} T^{n}=0 .
$$

Although we do not have a proof of this equation, it was discussed in [5] that the absolute values of the eigenvalues of $T$ are less than one. The existence of the smooth limit to the sliver state [4] also supports the existence of this limit, since the above discussion gives an analytical explanation for the equivalence between two definitions of the sliver, namely the original definition in [6] and the oscillator representation [5].

Up to a normalization, the wedge state over the sliver state is given by

$$
|n\rangle_{w}=\exp \left(-\frac{1}{2} s^{\dagger} C(-T)^{n-1} s^{\dagger}\right)|\Xi\rangle
$$




\subsection{Neumann Coefficients of $N$-string Vertex}

In this subsection, we will present a closed formula of the Neumann coefficients $V_{N}^{r s}$ in the $N$-string vertex

$$
\left|V_{N}\right\rangle=\exp \left(-\frac{1}{2} \sum_{r, s=1}^{N} a_{r}^{\dagger} V_{N}^{r s} a_{s}^{\dagger}\right)|0\rangle_{1 \cdots N}
$$

See 20] for the earlier discussion on the relation between the comma vertex and the Neumann coefficient.

We can easily generalize the comma representation of the 3 -string vertex (2.19) to the $N$-string vertex:

$$
\left|V_{N}\right\rangle=\exp \left(-\sum_{r=1}^{N} s_{r}^{\dagger} C L s_{r+1}^{\dagger}\right)|\Xi\rangle_{1 \cdots N}=\exp \left(-\frac{1}{2} \sum_{r, s=1}^{N} s_{r}^{\dagger} \widehat{V}_{N}^{r s} s_{s}^{\dagger}\right)|\Xi\rangle_{1 \cdots N},
$$

where $\widehat{V}_{N}^{r s}$ is given by

$$
C \widehat{V}_{N}=\left(\begin{array}{cccccc}
0 & L & 0 & 0 & \cdots & R \\
R & 0 & L & 0 & \cdots & 0 \\
0 & R & 0 & L & \cdots & 0 \\
& & \ddots & \ddots & \ddots & \\
0 & \cdots & 0 & R & 0 & L \\
L & 0 & \cdots & 0 & R & 0
\end{array}\right)
$$

in other words,

$$
C \widehat{V}_{N}^{r s}=\delta_{s-r, 1} L+\delta_{r-s, 1} R
$$

This expression of $N$-string vertex satisfies the required gluing relations [9]

$$
\begin{aligned}
& { }_{N}\left\langle I \mid V_{N}\right\rangle_{1 \cdots N}=\left|V_{N-1}\right\rangle_{1 \cdots N-1}, \\
& \left\langle R(K, L)|| V_{M+1}\right\rangle_{1 \cdots M, K}\left|V_{N+1}\right\rangle_{L, M+1, \cdots, M+N}=\left|V_{M+N}\right\rangle_{1 \cdots M+N} .
\end{aligned}
$$

By going back to the original variables, we find that $V_{N}^{r s}$ is given by

$$
C V_{N}=\left(T+C \widehat{V}_{N}\right)\left(1+C \widehat{V}_{N} T\right)^{-1}
$$

where $\left(T+C \widehat{V}_{N}\right)^{r s}=T \delta^{r s}+C \widehat{V}_{N}^{r s}$. For example, 4-string coefficient $V_{4}^{r s}$ becomes

$$
\begin{aligned}
& C V_{4}^{11}=-C V_{4}^{13}=\frac{T}{1+T^{2}}=\frac{X}{1+X}, \\
& C V_{4}^{12}=\frac{L+T^{2} R}{1+T^{2}}=\frac{1}{2}\left(1+\frac{Y-Z}{1+X}\right), \\
& C V_{4}^{14}=\frac{R+T^{2} L}{1+T^{2}}=\frac{1}{2}\left(1+\frac{Z-Y}{1+X}\right) .
\end{aligned}
$$

This expression agrees with the result in [17]. 
For general $N$, we can find the Neumann coefficients as follows. Since $V_{N}^{r s}$ depends only on $s-r \bmod N$, we can regard it as a vector. For the object $A_{s}$ with a $\bmod N$ index, we define the discrete Fourier transformation

$$
A_{s}=\frac{1}{N} \sum_{k=1}^{N} \omega^{k s} \widehat{A}_{k}
$$

where $\omega=\exp (2 \pi i / N)$. Then the convolution of two objects $A_{r}$ and $B_{s}$ becomes

$$
\sum_{r=1}^{N} A_{r} B_{s-r}=\frac{1}{N} \sum_{k=1}^{N} \omega^{k s} \widehat{A}_{k} \widehat{B}_{k}
$$

Using the Fourier representation of the matrices in the right-hand-side of (4.21)

$$
\begin{aligned}
\left(T+C \widehat{V}_{N}\right)^{r s} & =\frac{1}{N} \sum_{k=1}^{N} \omega^{k(s-r)}\left[\left(T+\omega^{-k}\right) L+\left(T+\omega^{k}\right) R\right] \\
{\left[\left(1+C \widehat{V}_{N} T\right)^{-1}\right]^{r s} } & =\frac{1}{N} \sum_{k=1}^{N} \omega^{k(s-r)}\left[\frac{1}{1+T \omega^{-k}} L+\frac{1}{1+T \omega^{k}} R\right]
\end{aligned}
$$

the Neumann coefficients for the general $N$ are found to be

$$
\begin{aligned}
C V_{N}^{r s} & =\frac{1}{N} \sum_{k=1}^{N} \omega^{k(s-r)}\left[\frac{T+\omega^{-k}}{1+T \omega^{-k}} L+\frac{T+\omega^{k}}{1+T \omega^{k}} R\right] \\
& =\frac{T(-T)^{[s-r]}+(-T)^{[s-r-1]}}{1-(-T)^{N}} L+\frac{T(-T)^{[r-s]}+(-T)^{[r-s-1]}}{1-(-T)^{N}} R
\end{aligned}
$$

where

$$
[s] \equiv s \bmod N, \quad[s] \in\{0,1, \cdots, N-1\} .
$$

One can check that this form of $V_{N}^{r s}$ satisfies the descent relation

$$
C V_{N-1}^{r s}=C V_{N}^{r s}+\frac{C V_{N}^{r N} C V_{N}^{N s}}{1-C V_{N}^{11}}
$$

which comes from (4.19). One can also check that

$$
C V_{N}^{r s}=C V_{2 N}^{r s}+C V_{2 N}^{r, s+N}=C V_{3 N}^{r s}+C V_{3 N}^{r, s+N}+C V_{3 N}^{r, s+2 N}=\cdots=\sum_{l=0}^{k-1} C V_{k N}^{r, s+l N}
$$

This is a generalization of the well-known relation between 3- and 6-string vertices [17].

Here we comment on the relation between the $N$-string vertices and the wedge states. The integral representation of Neumann coefficient is given by

$$
\left(V_{N}^{r s}\right)_{n m}=-\frac{1}{\sqrt{n m}} \oint \frac{d z}{2 \pi i} \oint \frac{d w}{2 \pi i} z^{-n} w^{-m} \frac{\partial_{z} f_{N}^{(r)}(z) \partial_{w} f_{N}^{(s)}(w)}{\left[f_{N}^{(r)}(z)-f_{N}^{(s)}(w)\right]^{2}}
$$


where

$$
f_{N}^{(r)}(z)=\tan \left(\frac{2}{N} \tan ^{-1} z-\frac{\pi r}{N}\right)=S^{r}\left(\tan \left(\frac{2}{N} \tan ^{-1} z\right)\right) .
$$

$S$ is an $S L(2, \mathbf{R})$ transformation

$$
S(z)=\frac{z \cos \frac{\pi}{N}-\sin \frac{\pi}{N}}{z \sin \frac{\pi}{N}+\cos \frac{\pi}{N}},
$$

which satisfies $S^{N}=1$. Since the matrix $C T_{N}$ in the wedge state $|N\rangle_{w}$ has the same integral representation as $V_{N}^{11}$, they should be the same. This can be also understood from the defining equations $|N\rangle_{w}=(|0\rangle)_{\star}^{N-1}={ }_{1}\langle 0| \cdots_{N-1}\left\langle 0|| V_{N}\right\rangle$. As expected, the diagonal component in (4.27) agrees with 4.8 )

$$
C V_{N}^{11}=T_{N}=\frac{T+(-T)^{N-1}}{1-(-T)^{N}}
$$

\section{$5 \quad$ Summary and Discussions}

In this paper, we analyzed the structure of the string field algebra using the so-called comma vertex. We obtained a closed formula of the $N$-string Neumann coefficient and showed that the diagonal element of this coefficient agrees with the width matrix of the wedge state.

It will be interesting to extend the idea of the comma vertex to the general CFT background. In fact, the 3 -string vertex over the sliver state was constructed in an arbitrary CFT [12]. Up to now, its relation to the comma vertex is unclear. It is also important to study the comma representation in the ghost sector. In [5], it was argued that the 3 -string vertex in the ghost sector can also be written in a comma form except for the subtlety associated with the ghost zero-modes.

\section{Acknowledgments}

We would like to thank N. Ishibashi, T. Kawano, T. Kugo, and B. Zwiebach for useful discussions. K. O. was supported in part by JSPS Research Fellowships for Young Scientists.

\section{References}

[1] E. Witten, "Noncommutative Geometry And String Field Theory," Nucl. Phys. B 268, 253 (1986).

[2] L. Rastelli, A. Sen and B. Zwiebach, "String field theory around the tachyon vacuum," hep-th/0012251.

[3] L. Rastelli, A. Sen and B. Zwiebach, "Vacuum String Field Theory," hep-th/0106010.

[4] L. Rastelli, A. Sen and B. Zwiebach, "Classical Solutions in String Field Theory around the Tachyon Vacuum," hep-th/0102112. 
[5] V. A. Kostelecky and R. Potting, "Analytical construction of a nonperturbative vacuum for the open bosonic string," Phys. Rev. D 63, 046007 (2001) hep-th/0008252.

[6] L. Rastelli and B. Zwiebach, "Tachyon potentials, star products and universality," hep-th/0006240.

[7] L. Rastelli, A. Sen and B. Zwiebach, "Half strings, projectors, and multiple D-branes in vacuum string field theory," hep-th/0105058.

[8] D. J. Gross and W. Taylor, "Split string field theory. I," hep-th/0105059; "Split string field theory. II," hep-th/0106036.

[9] T. Kawano and K. Okuyama, "Open String Fields As Matrices," JHEP 0106, 061 (2001) hep-th/0105129.

[10] L. Rastelli, A. Sen and B. Zwiebach, "Boundary CFT construction of D-branes in vacuum string field theory," hep-th/0105168.

[11] Y. Matsuo, "BCFT and sliver state," hep-th/0105175; "Identity projector and D-brane in string field theory," hep-th/0106027; "Projection operators and D-branes in purely cubic open string field theory," hep-th/0107007.

[12] J. R. David, "Excitations on wedge states and on the sliver," hep-th/0105184.

[13] I. Bars, "Map of Witten's * to Moyal's *," hep-th/0106157.

[14] J. Bordes, H. Chan, L. Nellen and S. T. Tsou, "Half String Oscillator Approach to String Field Theory," Nucl. Phys. B 351, 441 (1991).

[15] A. Abdurrahman and J. Bordes, "The relationship between the comma theory and Witten's string field theory. I," Phys. Rev. D 58, 086003 (1998).

[16] H. Chan and S. T. Tsou, "Yang-Mills Formulation Of Interacting Strings," Phys. Rev. D 39, 555 (1989); "String Theory Considered As A Local Gauge Theory Of An Extended Object," Phys. Rev. D 35, 2474 (1987).

[17] D. J. Gross and A. Jevicki, "Operator Formulation of Interacting String Field Theory (I)," Nucl. Phys. B 283, 1 (1987); "Operator Formulation of Interacting String Field Theory (II)," Nucl. Phys. B 287, 225 (1987).

[18] S. Samuel, "The Physical and Ghost Vertices in Witten's String Field Theory," Phys. Lett. B 181, 255 (1986).

[19] E. Cremmer, A. Schwimmer and C. Thorn, "The Vertex Function in Witten's Formulation of String Field Theory," Phys. Lett. B 179, 57 (1986).

[20] J. Bordes, A. Abdurrahman and F. Anton, "N string vertices in string field theory," Phys. Rev. D 49, 2966 (1994) hep-th/9306029. 